\title{
Dual Function Catalysts. Dehydrogenation and Asymmetric Intramolecular Diels-Alder Cycloaddition of N-Hydroxy Formate Esters and Hydroxamic Acids. Evidence for a Ruthenium-Acylnitroso Intermediate.
}

\author{
Chun P. Chow and Kenneth J. Shea* \\ Department of Chemistry \\ University of California, Irvine \\ Irvine, California, 92697-2025 \\ kjshea@uci.edu
}

\begin{abstract}
General. ${ }^{1} \mathrm{H}$ NMR and ${ }^{13} \mathrm{C}$ NMR spectra were recorded at ambient temperature at 500 $\mathrm{MHz}$ and $125 \mathrm{MHz}$, respectively, using a Bruker Omega 500 or GN 500 spectrometer. The data are reported as follows: chemical shift in ppm referenced to the residual proton(s) of $\mathrm{CDCl}_{3}$ or DMSO on the $\delta$ scale, multiplicity (br = broad, $\mathrm{s}=$ singlet, $\mathrm{d}=$ doublet, $\mathrm{t}=$ triplet, $\mathrm{q}=$ quartet, $\mathrm{m}=$ multiplet $)$, coupling constants $(\mathrm{Hz})$ and integration. High resolution mass spectra were acquired on a VG Analytical 7070E or Fisons Autospec spectrometer, and were obtained by peak matching. Optical rotations were measured using a JASCO DIP-370 digital polarimeter; concentration $c$ is reported in $\mathrm{g} / 100 \mathrm{~mL}$. Melting points are reported uncorrected. Analytical thin layer chromatography was performed using EM Reagents $0.25 \mathrm{~mm}$ silica gel $60-\mathrm{F}$ plates. Liquid chromatography was performed using forced flow (flash chromatography) of the indicated solvent system on EM Reagents silica gel $\left(\mathrm{SiO}_{2}\right) 60$ (230-400) mesh. All reactions were carried out under an atmosphere of nitrogen in glassware which had been flame-dried under a stream of nitrogen. Unless otherwise noted, all reagents were commercially obtained and, where appropriate, purified prior to use. THF, $\mathrm{Et}_{2} \mathrm{O}$, ethanol, and $\mathrm{CH}_{2} \mathrm{Cl}_{2}$ were dried by filtration through alumina according to the procedure of Grubbs (Pangborn, A. B.; Giardello, M. A.; Grubbs, R. H.; Rosen, R. K.; Timmers, F. J. Organometallics 1996, 15, 1518-1520). Methanol was distilled from a solution of magnesium turnings/magnesium methoxide under reflux. Acetone and $\mathrm{CHCl}_{3}$ were dried over 3 angstrom molecular sieves and isopropanol was dried over 5 angstrom molecular sieves.
\end{abstract}

Chiral HPLC. The enantiomeric excess of cycloadducts 3, 6, 9, and 12 was determined by HPLC using an analytical chiralcel OD-H column manufactured by Daicel Chemical Industries, Ltd. and is reported as an average \% ee from triplicate injections. Each elution was performed with an injection volume of 10 microliter eluting with 5\% 2-propanol in $n$-hexane at $25{ }^{\circ} \mathrm{C}$. Both enantiomers of each cycloadduct were separated and detected at either $210 \mathrm{~nm}$ or $254 \mathrm{~nm}$. The retention times (from the solvent peak) of the enantiomers are 6.6 and $6.8 \mathrm{~min}$ for 3,11.0 and $12.1 \mathrm{~min}$ for $\mathbf{6}, 9.9$ and $10.4 \mathrm{~min}$ for $\mathbf{9}$, and 13.7 and $14.0 \mathrm{~min}$ for $\mathbf{1 2}$. 
Supporting Data - Chow and Shea

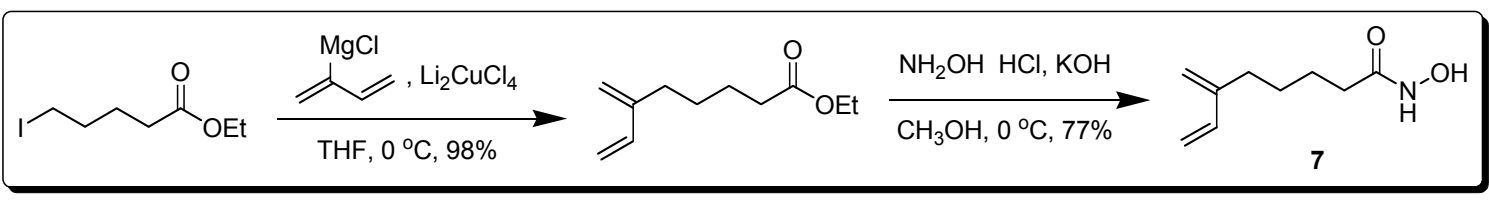<smiles>CCOC(=O)CCCCI</smiles>

Ethyl 5-iodopentanoate: Sodium iodide (4.66 g, $31.1 \mathrm{mmol})$ was added to $20 \mathrm{~mL}$ anhydrous acetone and brought to gentle reflux, effecting solution. To this stirred solution was added ethyl 4-bromovalerate (5.00 g, $23.9 \mathrm{mmol})$ dropwise over $1 \mathrm{~h}$. Reflux was continued for $4 \mathrm{~h}$ or until no further precipitation was observed. The mixture was cooled, diluted with equal volume of ethyl ether, filtered and evaporated in vacuo to an oil. The oil was taken up in $40 \mathrm{~mL}$ ethyl ether and washed twice with $12 \mathrm{~mL}$ poritions of $2 \% \mathrm{NaOH}$, water, and brine, and dried over $\mathrm{MgSO}_{4}$, yield $5.88 \mathrm{~g}(96 \%)$ of ethyl 5-iodopentanoate. This product was used directly in the next step without purification. ${ }^{1} \mathrm{H} \mathrm{NMR}\left(\mathrm{CDCl}_{3}\right) \delta 4.11(\mathrm{q}, J=7.1 \mathrm{~Hz}, 2 \mathrm{H}), 3.18(\mathrm{t}, J=6.9 \mathrm{~Hz}, 2 \mathrm{H}), 2.31$ (t, $J=7.3 \mathrm{~Hz}, 2 \mathrm{H}$ ), 1.85 (dddd, $J=7.2,7.2,7.2,7.2 \mathrm{~Hz}, 2 \mathrm{H}$ ), 1.72 (dddd, $J=7.2,7.2$, 7.2, $7.2 \mathrm{~Hz}, 2 \mathrm{H}), 1.24(\mathrm{t}, J=7.1 \mathrm{~Hz}, 3 \mathrm{H}) ;{ }^{13} \mathrm{C} \mathrm{NMR}\left(\mathrm{CDCl}_{3}\right) \delta 173.3,60.6,33.3,32.9$, 26.0, 14.4, 6.1. IR (NaCl film) 2979, 2937, 1732, 1447, 1374, $1188 \mathrm{~cm}^{-1}$. HRMS (EI) calculated for $\mathrm{C}_{7} \mathrm{H}_{14} \mathrm{IO}_{2}(\mathrm{M}+\mathrm{H})^{+}$257.0039, observed 257.0034 .<smiles>C=CC(=C)CCCCC(=O)OCC</smiles>

iodopentanoate $(8.03 \mathrm{~g}, 31.4 \mathrm{mmol})$ and $\mathrm{Li}_{2} \mathrm{CuCl}_{4}(1.9 \mathrm{~mL}$ of a $0.5 \mathrm{M}$ solution, 0.94 $\mathrm{mmol})$ in THF $(16 \mathrm{~mL})$ at $0{ }^{\circ} \mathrm{C}$ was added a solution of chloroprene Grignard $(35 \mathrm{~mL}$ of a $0.91 \mathrm{M}$ solution, $31.4 \mathrm{mmol}$ ) dropwise. The reaction mixture was stirred at $0{ }^{\circ} \mathrm{C}$ for $1 \mathrm{~h}$ then warmed to room temperature and stirred for $4 \mathrm{~h}$. A solution of saturated aqueous $\mathrm{NH}_{4} \mathrm{Cl}(20 \mathrm{~mL})$ and water $(15 \mathrm{~mL})$ was added to the reaction mixture. The mixture was extracted with ether $(2 \times 50 \mathrm{~mL})$, dried $\left(\mathrm{MgSO}_{4}\right)$, and concentrated in vacuo to afford diene 24 as a pale yellow oil $(5.61 \mathrm{~g}, 98 \%):{ }^{1} \mathrm{H}$ NMR $\left(\mathrm{CDCl}_{3}\right) \delta 6.35(\mathrm{dd}, J=17.6,10.8$ $\mathrm{Hz}, 1 \mathrm{H}), 5.21(\mathrm{~d}, J=17.6 \mathrm{~Hz}, 1 \mathrm{H}), 5.05(\mathrm{~d}, J=10.8 \mathrm{~Hz}, 1 \mathrm{H}), 5.01(\mathrm{~s}, 1 \mathrm{H}), 4.98(\mathrm{~s}, 1$ $\mathrm{H}), 3.66(\mathrm{~s}, 3 \mathrm{H}), 2.33(\mathrm{t}, J=7.3 \mathrm{~Hz}, 2 \mathrm{H}), 2.22(\mathrm{t}, J=7.8 \mathrm{~Hz}, 2 \mathrm{H}), 1.67$ (quintet, $J=7.4$ $\mathrm{Hz}, 2 \mathrm{H}), 1.56-1.49(\mathrm{~m}, 2 \mathrm{H}) ;{ }^{13} \mathrm{C} \mathrm{NMR}\left(\mathrm{CDCl}_{3}\right) \delta 174.1,145.9,138.8,115.7,113.1$, 51.4, 33.9, 31.0, 27.6, 24.8; IR ( $\mathrm{NaCl}$ film) 3089, 2949, 1738, 1436, $1198 \mathrm{~cm}^{-1}$; HRMS (EI) calculated for $\mathrm{C}_{10} \mathrm{H}_{16} \mathrm{O}_{2}(\mathrm{M})^{+} 168.1150$, observed 168.1149 .<smiles>C=CC(=C)CCCCC(=O)NO</smiles>

7

N-Hydroxy-6-methylene-7-octenamide (7): To a solution of hydroxylamine hydrochloride $(24.8 \mathrm{~g}, 38.4 \mathrm{mmol})$ in $\mathrm{MeOH}(75 \mathrm{~mL})$ at $0{ }^{\circ} \mathrm{C}$ was added a 


\section{Supporting Data - Chow and Shea}

solution of $\mathrm{KOH}(43.1 \mathrm{~g}, 768 \mathrm{mmol})$ in $\mathrm{MeOH}(150 \mathrm{~mL})$ dropwise. The solution was stirred 20 min after the addition was complete. Ethyl 6-methyleneoct-7-enoate $(7.00 \mathrm{~g}$, $38.4 \mathrm{mmol})$ dissolved in $\mathrm{MeOH}(40 \mathrm{~mL})$ was added dropwise and the white solution was stirred overnight. Water $(100 \mathrm{~mL})$ was added and the $\mathrm{pH}$ was adjusted to 6 with concentrated $\mathrm{HCl}$. The mixture was extracted with $\mathrm{CHCl}_{3}(3 \times 650 \mathrm{~mL})$ and the extracts were dried $\left(\mathrm{MgSO}_{4}\right)$ and concentrated. The residue was chromatographed on silica gel eluting with $\mathrm{CH}_{2} \mathrm{Cl}_{2} / \mathrm{MeOH}(95: 5)$ to afford $5.35 \mathrm{~g} \mathrm{(76 \% )}$ of hydroxamic acid 7 as a pale yellow oil. ${ }^{1} \mathrm{H}$ NMR $\left(\mathrm{CDCl}_{3}\right) \delta 9.08$ (br s, $\left.1 \mathrm{H}\right), 6.33$ (dd, $\left.J=17.6,10.8 \mathrm{~Hz}, 1 \mathrm{H}\right), 5.18$ (d, $J=17.6 \mathrm{~Hz}, 1 \mathrm{H}), 5.03(\mathrm{~d}, J=10.8 \mathrm{~Hz}, 1 \mathrm{H}), 4.99(\mathrm{~s}, 1 \mathrm{H}), 4.96(\mathrm{~s}, 1 \mathrm{H}), 2.19(\mathrm{t}, J=7.5$ $\mathrm{Hz}, 2 \mathrm{H}$ ), 2.15 (t, $J=7.5 \mathrm{~Hz}, 2 \mathrm{H}$ ), 1.65 (quintet, $J=7.6 \mathrm{~Hz}, 2 \mathrm{H}$ ), 1.49 (quintet, $J=7.6$ $\mathrm{Hz}, 2 \mathrm{H}) ;{ }^{13} \mathrm{C} \mathrm{NMR}\left(\mathrm{CDCl}_{3}\right) \delta$ 171.6, 145.7, 138.8, 115.9, 113.2, 32.8, 30.9, 27.5, 25.2; IR (NaCl film) 3197 (br), 2937, 1635, $1540 \mathrm{~cm}^{-1}$; HRMS (CI) calculated for $\mathrm{C}_{9} \mathrm{H}_{16} \mathrm{NO}_{2}$ $(\mathrm{M}+\mathrm{H})^{+}$170.1181, observed 170.1184 .

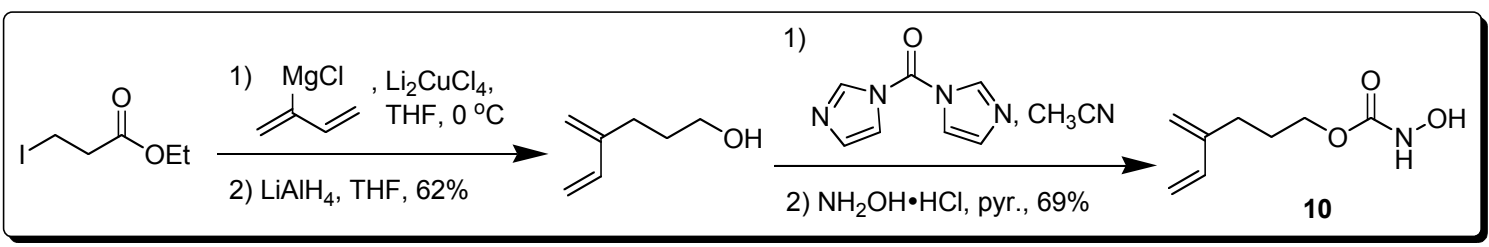<smiles>CCOC(=O)CCI</smiles>

Ethyl 3-iodopropanoate: Sodium iodide (15.23 g, $101.6 \mathrm{mmol})$ was added to $60 \mathrm{~mL}$ anhydrous acetone and brought to gentle reflux, effecting solution. To this stirred solution was added ethyl 3-bromopropionate (14.15 g, $78.16 \mathrm{mmol})$ dropwise over $1 \mathrm{~h}$. Reflux was continued for $4 \mathrm{~h}$ or until no further precipitation was observed. The mixture was cooled, diluted with equal volume of ethyl ether, filtered and evaporated in vacuo to an oil. The oil was taken up in $100 \mathrm{~mL}$ ethyl ether and washed twice with $40 \mathrm{~mL}$ poritions of $2 \% \mathrm{NaOH}$, water, and brine, and dried over $\mathrm{MgSO}_{4}$, yield $17.48 \mathrm{~g}(98 \%)$ of ethyl 3-iodopropanoate. This product was used directly in the next step without purification. ${ }^{1} \mathrm{H} \mathrm{NMR}\left(\mathrm{CDCl}_{3}\right) \delta 4.18(\mathrm{q}, J=7.1 \mathrm{~Hz}, 2 \mathrm{H}), 3.33(\mathrm{t}, J=7.1 \mathrm{~Hz}, 2$ $\mathrm{H}), 2.96(\mathrm{t}, J=7.1 \mathrm{~Hz}, 2 \mathrm{H}), 1.28(\mathrm{t}, J=7.1 \mathrm{~Hz}, 3 \mathrm{H}) ;{ }^{13} \mathrm{C} \mathrm{NMR}\left(\mathrm{CDCl}_{3}\right) \delta 171.4,61.2$, $38.3,14.4,-3.47$. IR ( $\mathrm{NaCl}$ film) 2979, 2940, 1735, 1449, $1372 \mathrm{~cm}^{-1}$.

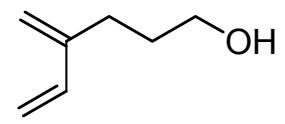

4-Methylenehex-5-en-1-ol: To a solution of $0.5 \mathrm{M} \mathrm{Li}_{2} \mathrm{CuCl}_{4}$ in THF (4.6 mL, $2.30 \mathrm{mmol})$ in THF $(37 \mathrm{~mL})$ at $0{ }^{\circ} \mathrm{C}$ was added ethyl 5-iodopropanoate. To the stirred solution was added slowly a $1.0 \mathrm{M}$ solution of chloroprene Grignard $(76.7 \mathrm{~mL}$, $76.7 \mathrm{mmol})$. The reaction was stirred $1 \mathrm{~h}$ at room temperature and needle stocked slowly into a stirred suspension of $\mathrm{LiAlH}_{4}(3.40 \mathrm{~g}, 89.7 \mathrm{mmol})$ in THF $(120 \mathrm{~mL})$. The reaction mixture was stirred $2 \mathrm{~h}$ at room temperature then worked up by slow addition of $\mathrm{H}_{2} \mathrm{O}(3.9$ $\mathrm{mL}), 15 \% \mathrm{NaOH}(3.5 \mathrm{~mL})$, and $\mathrm{H}_{2} \mathrm{O}(9.9 \mathrm{~mL})$. CAUTION: The reaction becomes highly 


\section{Supporting Data - Chow and Shea}

exothermic upon addition of $\mathrm{H}_{2} \mathrm{O}$ ! The resultant powder was filtered and washed three times with $\mathrm{Et}_{2} \mathrm{O}(3 \times 250 \mathrm{~mL})$. The organic extracts were combined, washed with brine, and dried over $\mathrm{MgSO}_{4}$. Purification by flash column chromatography on silica gel eluting with petroleum ether/ $\mathrm{Et}_{2} \mathrm{O}(60: 40)$ afforded $5.33 \mathrm{~g} \mathrm{(62 \% )}$ of 4-methylenehex-5-en-1-ol as a pale yellow oil. ${ }^{1} \mathrm{H}$ NMR $\left(\mathrm{CDCl}_{3}\right) \delta 6.38(\mathrm{dd}, J=17.6,10.8 \mathrm{~Hz}, 1 \mathrm{H}), 5.25(\mathrm{~d}, J=17.6$ $\mathrm{Hz}, 1 \mathrm{H}), 5.07$ (d, $J=10.9 \mathrm{~Hz}, 1 \mathrm{H}), 5.03(\mathrm{~s}, 1 \mathrm{H}), 5.02(\mathrm{~s}, 1 \mathrm{H}), 3.68$ (dd, $J=11.6,6.3$ $\mathrm{Hz}, 2 \mathrm{H}), 2.31(\mathrm{td}, J=7.7,0.8 \mathrm{~Hz}, 2 \mathrm{H}), 1.77$ (quintet, $J=6.4,7.8 \mathrm{~Hz}, 2 \mathrm{H}), 1.41(\mathrm{t}, J=$ $5.2 \mathrm{~Hz}, 1 \mathrm{H}) ;{ }^{13} \mathrm{C}$ NMR $\left(\mathrm{CDCl}_{3}\right) \delta 146.0,138.9,116.2,113.6,62.9,31.3,27.8$; IR $(\mathrm{NaCl}$ film) 3388 (br), 3089, 2942, 2873, $1060 \mathrm{~cm}^{-1}$; HRMS (EI) calculated for $\mathrm{C}_{7} \mathrm{H}_{12} \mathrm{O}(\mathrm{M})^{+}$ 112.0888, observed 112.0886 .

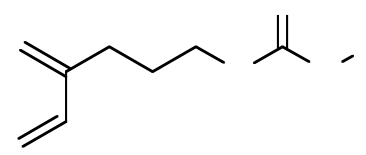

4-Methylene-4-pentene $\boldsymbol{N}$-hydroxycarbamate (10): To a solution of 4-methylenehex-5-en-1-ol $(0.80 \mathrm{~g}, 7.13 \mathrm{mmol})$ in $\mathrm{CH}_{3} \mathrm{CN}(24 \mathrm{~mL})$ was added $1,1^{\prime}$-carbonyldiimidazole $(3.47 \mathrm{~g}, 21.4 \mathrm{mmol})$. The solution was stirred $90 \mathrm{~min}$ then diluted with EtOAc $(105 \mathrm{~mL})$ and washed with water $(140 \mathrm{~mL})$. The organic layer was dried $\left(\mathrm{MgSO}_{4}\right)$ and concentrated to an oil. The oil was dissolved in pyridine $(10.5 \mathrm{~mL})$ and added to a solution of hydroxylamine hydrochloride $(1.38 \mathrm{~g}, 21.4 \mathrm{mmol})$ in pyridine $(13.3 \mathrm{~mL})$. The solution was stirred $2 \mathrm{~h}$, then diluted with EtOAc $(70 \mathrm{~mL})$ and washed with $10 \% \mathrm{HCl}(70 \mathrm{~mL})$. The organic extract was dried $\left(\mathrm{MgSO}_{4}\right)$, concentrated and chromatographed on silica gel eluting with $\mathrm{CHCl}_{3} / \mathrm{CH}_{3} \mathrm{OH}$ (99:01) to afford $0.89 \mathrm{~g} \mathrm{(73 \% )}$ of N-hydroxy formate ester 10 as a colorless oil. ${ }^{1} \mathrm{H}$ NMR (DMSO-d $\left.\mathrm{d}_{6}\right) \delta 9.54(\mathrm{br} \mathrm{s}, 1 \mathrm{H})$, 6.38 (dd, $J=17.6,10.8 \mathrm{~Hz}, 1 \mathrm{H}), 5.25(\mathrm{~d}, J=17.6 \mathrm{~Hz}, 1 \mathrm{H}), 5.09(\mathrm{~d}, J=10.8 \mathrm{~Hz}, 1 \mathrm{H})$, $5.07(\mathrm{~s}, 1 \mathrm{H}), 5.03(\mathrm{~s}, 1 \mathrm{H}), 4.00(\mathrm{t}, J=6.6 \mathrm{~Hz}, 2 \mathrm{H}), 2.22(\mathrm{t}, J=7.7 \mathrm{~Hz}, 2 \mathrm{H}), 1.71$ (quintet, $J=7.5,6.6 \mathrm{~Hz}, 2 \mathrm{H}$ ); ${ }^{13} \mathrm{C}$ NMR $\left(\right.$ DMSO-d $_{6}$ ) $\delta 157.9,145.1,138.5,116.4,113.9$, 63.6, 27.2, 26.8; IR (NaCl film) 3298, 3089, 2959, 1718, 1595, $1466 \mathrm{~cm}^{-1}$; HRMS (CI) calculated for $\mathrm{C}_{8} \mathrm{H}_{14} \mathrm{NO}_{3}(\mathrm{M})^{+}$172.0974, observed 172.0973.

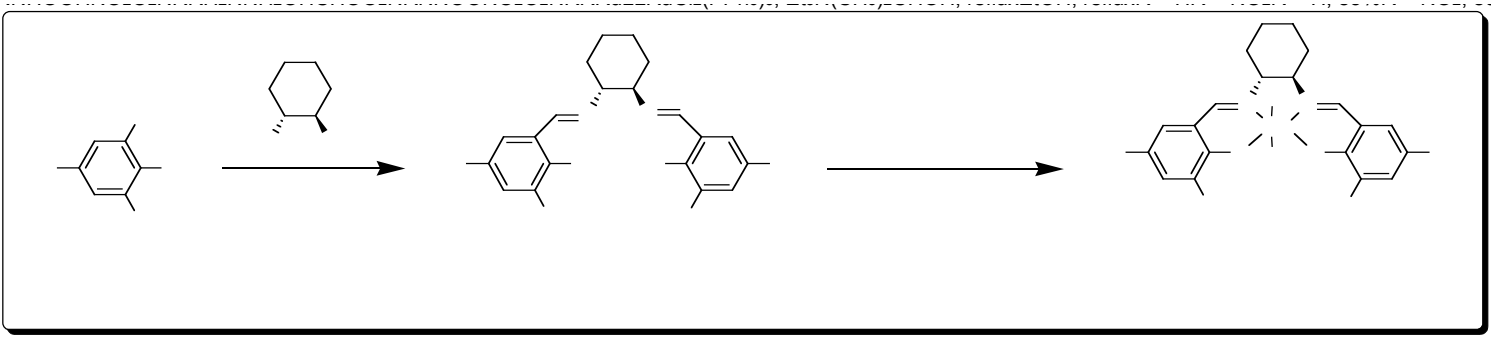

General procedure for salen ligand preparation: To a solution of $(R, R)-1,2-$ diaminocyclohexane $(2.2 \mathrm{mmol})$ in ethanol $(22 \mathrm{~mL})$ stirred under reflux was added a solution of substituted salicylaldehyde $(2.2 \mathrm{mmol})$ in ethanol $(22 \mathrm{~mL})$ dropwise over a period of 30 minutes. The reaction mixture was stirred $2 \mathrm{~h}$ under reflux. After the 


\section{Supporting Data - Chow and Shea}

resulting mixture was cooled to room temperature, the precipitate was collected by filtration and washed with ethanol $(11 \mathrm{~mL})$. In the reaction where no precipitate was formed, $\mathrm{H}_{2} \mathrm{O}(11 \mathrm{~mL})$ was added to induce precipitation. The product was allowed to air dried overnight and was obtained as a yellow or orange powder.

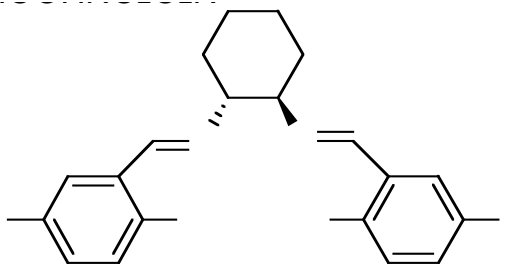

$(R, R$ ) -N,N'-Bis(5-nitro-salicylidene)-1,2cyclohexanediamine: $\operatorname{mp} 218-220{ }^{\circ} \mathrm{C} ;[\alpha]^{20}{ }_{\mathrm{D}}=-20.7 \quad\left(c 1.00, \mathrm{CH}_{2} \mathrm{Cl}_{2}\right) ;{ }^{1} \mathrm{H}$ NMR $\left(\mathrm{CDCl}_{3}\right) \delta 14.27$ (br s, $\left.2 \mathrm{H}\right), 8.35(\mathrm{~s}, 2 \mathrm{H}), 8.15(\mathrm{~m}, 4 \mathrm{H}), 6.96(\mathrm{~d}, J=9.9 \mathrm{~Hz}, 2 \mathrm{H}), 3.46$ $(\mathrm{m}, 2 \mathrm{H}), 2.05-1.50(\mathrm{~m}, 8 \mathrm{H}) ;{ }^{13} \mathrm{C} \mathrm{NMR}\left(\mathrm{CDCl}_{3}\right) \delta 167.5,163.9,139.3,128.4,128.1$, 118.6, 117.3, 72.2, 32.9, 24.2; HRMS (CI) calculated for $\mathrm{C}_{20} \mathrm{H}_{20} \mathrm{~N}_{4} \mathrm{O}_{6}(\mathrm{M})^{+}$412.1383, observed 412.1385 .

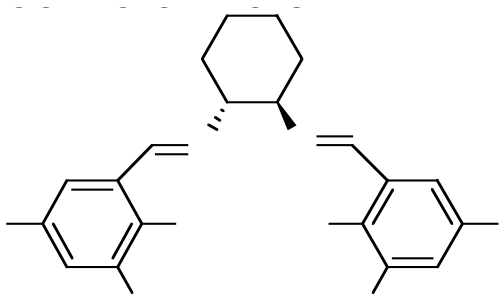

$(R, R)-N, N^{\prime}$-Bis(3,5-dinitro-salicylidene)-

1,2-cyclohexanediamine: $\operatorname{mp} 251{ }^{\circ} \mathrm{C} ;[\alpha]^{20}{ }_{\mathrm{D}}=-155{ }^{\circ} \mathrm{C}(c 1.00$, DMSO $) ;{ }^{1} \mathrm{H}$ NMR $\left(\mathrm{DMSO}_{-} \mathrm{d}_{6}\right) \delta 13.55$ (br s, $\left.2 \mathrm{H}\right), 8.91$ (s, $\left.2 \mathrm{H}\right), 8.71-8.64(\mathrm{~m}, 4 \mathrm{H}), 4.24(\mathrm{~d}, J=9.4 \mathrm{~Hz}, 2$ H), $2.15(\mathrm{~d}, J=11.1 \mathrm{~Hz}, 2 \mathrm{H}), 1.85(\mathrm{t}, J=7.8 \mathrm{~Hz}, 4 \mathrm{H}), 1.39(\mathrm{t}, J=9.7 \mathrm{~Hz}, 2 \mathrm{H}) ;{ }^{13} \mathrm{C}$ NMR (DMSO-d $)_{6} \delta 169.7,167.8,140.7,137.5,130.0,127.4,117.0,63.3,30.7,23.4$; HRMS (ESI) calculated for $\mathrm{C}_{20} \mathrm{H}_{18} \mathrm{NaN}_{6} \mathrm{O}_{10}(\mathrm{M}+\mathrm{Na})^{+}$525.0982, observed 525.0999.

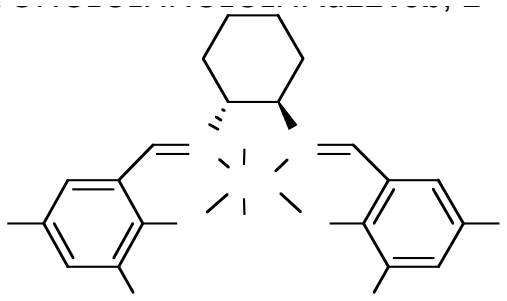

Dinitroso ruthenium complex (13b): To a solution of $(R, R)-N, N$ '-bis(3,5-dinitro-salicylidene)-1,2-cyclohexanediamine $(0.20 \mathrm{~g}, 0.40$ $\mathrm{mmol})$ and tris(triphenylphosphine) ruthenium(II) chloride $(0.36 \mathrm{~g}, 0.38 \mathrm{mmol})$ in isopropanol $(25 \mathrm{~mL})$ was added triethylamine $(0.32 \mathrm{~mL}, 6$ equiv). The reaction mixture was stirred $2 \mathrm{~h}$ under reflux. After the reaction mixture was cooled, the precipitate was collected by filtration and recrystallized from hexanes: $\mathrm{CH}_{2} \mathrm{Cl}_{2}(60: 10)$ to afford $0.30 \mathrm{~g}$ 


\section{Supporting Data - Chow and Shea}

(71\%) of complex 13b as a brown solid. mp $>250{ }^{\circ} \mathrm{C} ;{ }^{1} \mathrm{H}$ NMR (acetone-d $\left.\mathrm{d}_{6}\right) \delta 8.25$ (s, 2 H), $7.58(\mathrm{dd}, J=9.5,2.9 \mathrm{~Hz}, 2 \mathrm{H}), 7.37-7.08(\mathrm{~m}, 32 \mathrm{H}), 6.27$ (d, $J=9.5 \mathrm{~Hz}, 2 \mathrm{H}), 2.62$ $(\mathrm{m}, 2 \mathrm{H}), 1.9-0.9$ (m, $8 \mathrm{H}) ;{ }^{13} \mathrm{C}$ NMR (acetone-d $\left.{ }_{6}\right) \delta 173.5,156.9,134.9-122.2(\mathrm{~m}), 73.9$, 28.8, 24.7; Anal. Calcd for $\mathrm{C}_{56} \mathrm{H}_{46} \mathrm{~N}_{6} \mathrm{O}_{10} \mathrm{P}_{2} \mathrm{Ru}$ : C, 59.73; H 4.12. Found C, 59.85; H 4.15.

Dehydrogenation and Diels-Alder cycloaddition of $\mathrm{N}$-hydroxy formate esters and hydroxamic acids with chiral ruthenium complex $13 \mathrm{~b}$.

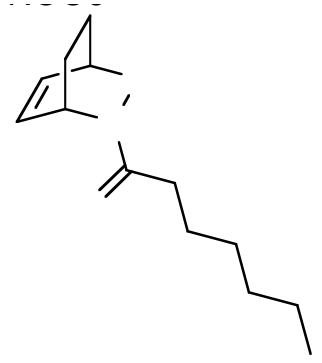

1-(3-Oxa-2-aza-bicyclo[2.2.2]oct-5-en-2-yl)heptan-1-one (3): To a solution of heptanohydroxamic acid $(1,0.10 \mathrm{~g}, 0.63 \mathrm{mmol})$ and ruthenium complex (13b, $\left.0.7 \mathrm{mg}, 0.63 \times 10^{-3} \mathrm{mmol}\right)$ in $\mathrm{CH}_{2} \mathrm{Cl}_{2}(1.7 \mathrm{~mL})$ was added 1,3-cyclohexadiene $(50.3 \mathrm{mg}, 0.63 \mathrm{mmol})$ and $5.3 \mathrm{M}{ }^{t} \mathrm{BOOH}$ in decane $(119 \mu \mathrm{L})$. The reaction mixture was stirred $1 \mathrm{~h}$ at room temperature. The reaction mixture was washed two times with $\mathrm{H}_{2} \mathrm{O}(2$ x $8 \mathrm{~mL}$ ) and brine $(8 \mathrm{~mL})$, and dried over $\mathrm{MgSO}_{4}$. The crude mixture was purified by silica gel chromatography eluting with $\mathrm{CH}_{3} \mathrm{Cl}_{3}: \mathrm{CH}_{3} \mathrm{OH}(98.5: 1.5)$ to afford $72 \mathrm{mg}(48 \%)$ of cycloadduct 3 as a light orange oil. ${ }^{1} \mathrm{H} \mathrm{NMR}\left(\mathrm{CDCl}_{3}\right) \delta 6.62(\mathrm{t}, J=7.4 \mathrm{~Hz}, 1 \mathrm{H}), 6.50$ $(\mathrm{t}, J=6.2 \mathrm{~Hz}, 1 \mathrm{H}), 5.26(\mathrm{~s}, 1 \mathrm{H}), 4.73(\mathrm{~s}, 1 \mathrm{H}), 2.16(\mathrm{~m}, 4 \mathrm{H}), 1.28-0.85(\mathrm{~m}, 13 \mathrm{H}) ;{ }^{13} \mathrm{C}$ $\operatorname{NMR}\left(\mathrm{CDCl}_{3}\right) \delta 173.3,133.3,131.3,71.9,46.5,33.4,31.7,29.2,24.3,23.8,22.7,21.2$, 14.2; $\delta$ IR (NaCl film) 2931, 2859, 1651, 1456, $1364 \mathrm{~cm}^{-1}$; HRMS (CI) calculated for $\mathrm{C}_{13} \mathrm{H}_{22} \mathrm{NO}_{2}(\mathrm{M}+\mathrm{H})^{+}$224.1651, observed 224.1655.

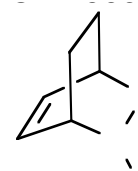

tert-Butyl 3-oxa-2-aza-bicyclo[2.2.2] oct-5-ene-2-carboxylate (6): To a solution of tert-butyl $N$-hydroxycarbamate $(4,0.20 \mathrm{~g}, 1.51 \mathrm{mmol})$ and ruthenium complex $\left(13 \mathrm{~b}, 1.6 \mathrm{mg}, 1.51 \times 10^{-3} \mathrm{mmol}\right)$ in $\mathrm{CH}_{2} \mathrm{Cl}_{2}(4.0 \mathrm{~mL})$ was added $1,3-$ cyclohexadiene $(120 \mathrm{mg}, 1.50 \mathrm{mmol})$ and $5.3 \mathrm{M}{ }^{t} \mathrm{BOOH}$ in decane $(0.28 \mathrm{~mL})$. The reaction mixture was stirred $1 \mathrm{~h}$ at room temperature. The reaction mixture was washed two times with $\mathrm{H}_{2} \mathrm{O}(2 \times 20 \mathrm{~mL})$ and brine $(20 \mathrm{~mL})$, and dried over $\mathrm{MgSO}_{4}$. The crude mixture was purified by silica gel chromatography eluting with $\mathrm{CH}_{3} \mathrm{Cl}_{3}: \mathrm{CH}_{3} \mathrm{OH}$ (98:02) to afford $0.26 \mathrm{~g}(82 \%)$ of cycloadduct 6 as a yellow oil. ${ }^{1} \mathrm{H}$ NMR $\left(\mathrm{CDCl}_{3}\right) \delta 6.53(\mathrm{~m}, 2$ $\mathrm{H}), 4.71(\mathrm{~m}, 2 \mathrm{H}), 2.17(\mathrm{~m}, 1 \mathrm{H}), 2.08(\mathrm{~m}, 1 \mathrm{H}), 1.47(\mathrm{~m}, 1 \mathrm{H}), 1.45(\mathrm{~s}, 9 \mathrm{H}), 1.34(\mathrm{~m}, 1$ $\mathrm{H}) ;{ }^{13} \mathrm{C} \mathrm{NMR}\left(\mathrm{CDCl}_{3}\right) \delta 157.9,131.9,131.7,81.7,70.8,50.3,34.4,28.4,23.8,20.7$; LRMS (EI) calculated for $\mathrm{C}_{11} \mathrm{H}_{17} \mathrm{NO}_{3}(\mathrm{M})^{+} 211.12$, observed 211.15. 


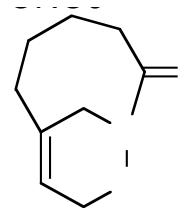

10-Oxa-1-aza-bicyclo[5.3.1] undec-7-en-2-one (9): To a solution of $N$ hydroxy-6-methylene-7-octenamide $(\mathbf{7}, 0.15 \mathrm{~g}, 0.89 \mathrm{mmol})$ and ruthenium complex $(\mathbf{1 3 b}$, $\left.10.0 \mathrm{mg}, 0.89 \times 10^{-3} \mathrm{mmol}\right)$ in $\mathrm{CH}_{2} \mathrm{Cl}_{2}(2.4 \mathrm{~mL})$ was added $5.3 \mathrm{M}^{t} \mathrm{BOOH}$ in decane (167 $\mu \mathrm{L})$. The reaction mixture was stirred $1 \mathrm{~h}$ at room temperature. The reaction mixture was washed two times with $\mathrm{H}_{2} \mathrm{O}(2 \times 10 \mathrm{~mL})$ and brine $(10 \mathrm{~mL})$, and dried over $\mathrm{MgSO}_{4}$. The crude mixture was purified by silica gel chromatography eluting with $\mathrm{CH}_{3} \mathrm{Cl}_{3}$ :EtOAc (95:05) to afford $91 \mathrm{mg}(61 \%)$ of cycloadduct 9 as a white solid. ${ }^{1} \mathrm{H}$ NMR $\left(\mathrm{CDCl}_{3}\right) \delta 5.47$ $(\mathrm{m}, 1 \mathrm{H}), 4.78$ (dddd, $J=15.0,3.8,2.0,2.0 \mathrm{~Hz}, 1 \mathrm{H}), 4.36$ (dddd, $J=15.0,4.1,2.0,2.0$ $\mathrm{Hz}, 1 \mathrm{H}), 3.96$ (d, $J=16.3 \mathrm{~Hz}, 1 \mathrm{H}), 3.66$ (br d, $J=16.3 \mathrm{~Hz}, 1 \mathrm{H}), 2.48-2.42$ (m, $2 \mathrm{H})$, $2.19(\mathrm{t}, J=12.0 \mathrm{~Hz}, 1 \mathrm{H}), 2.07-1.99(\mathrm{~m}, 3 \mathrm{H}), 1.78-1.69(\mathrm{~m}, 1 \mathrm{H}), 1.39-1.30(\mathrm{~m}, 1 \mathrm{H})$; ${ }^{13} \mathrm{C} \mathrm{NMR}\left(\mathrm{CDCl}_{3}\right) \delta 179.9,139.7,120.8,67.0,48.0,36.5,33.5,26.6,24.2 ; \mathrm{IR}(\mathrm{KBr})$ 2944, 2854, 1692, 1444, 1329, $1160 \mathrm{~cm}^{-1}$; HRMS (CI) calculated for $\mathrm{C}_{9} \mathrm{H}_{14} \mathrm{NO}_{2}(\mathrm{M}+\mathrm{H})^{+}$ 168.1024, observed 168.1017.

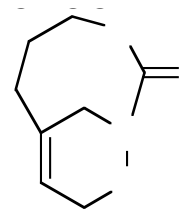

3,10-Dioxa-1-aza-bicyclo[5.3.1] undec-7-en-2-one (12): To a solution of 4-methylene-4-pentene $N$-hydroxycarbamate $(\mathbf{1 0}, 0.10 \mathrm{~g}, 0.58 \mathrm{mmol})$ and ruthenium complex (13b, $\left.6.0 \mathrm{mg}, 0.58 \times 10^{-3} \mathrm{mmol}\right)$ in $\mathrm{CH}_{2} \mathrm{Cl}_{2}(1.6 \mathrm{~mL})$ was added $5.3 \mathrm{M}^{t} \mathrm{BOOH}$ in decane $(110 \mu \mathrm{L})$. The reaction mixture was stirred $1 \mathrm{~h}$ at room temperature. The reaction mixture was washed two times with $\mathrm{H}_{2} \mathrm{O}(2 \times 7.7 \mathrm{~mL})$ and brine $(7.7 \mathrm{~mL})$, and dried over $\mathrm{MgSO}_{4}$. The crude mixture was purified by silica gel chromatography eluting with $\mathrm{CH}_{3} \mathrm{Cl}_{3}$ :EtOAc (95:05) to afford $64 \mathrm{mg}(43 \%)$ of cycloadduct 12 as a yellow oil. ${ }^{1} \mathrm{H}$ $\operatorname{NMR}\left(\mathrm{CDCl}_{3}\right) \delta 5.76(\mathrm{~m}, 1 \mathrm{H}), 4.72(\mathrm{ddt}, J=14.3,5.5,1.1 \mathrm{~Hz}, 1 \mathrm{H}), 4.40$ (ddddd, $J=$ $14.3,3.9,3.9,1.9,1.9 \mathrm{~Hz}, 1 \mathrm{H}), 4.36(\mathrm{~m}, 1 \mathrm{H}), 4.20(\mathrm{dd}, J=15.0,2.1 \mathrm{~Hz}, 1 \mathrm{H}), 4.07$ (m, $1 \mathrm{H}), 3.48$ (ddd, $J=15.0,3.1,3.1 \mathrm{~Hz}, 1 \mathrm{H}), 2.48(\mathrm{~m}, 1 \mathrm{H}), 2.25(\mathrm{~m}, 2 \mathrm{H}), 1.89(\mathrm{~m}, 1 \mathrm{H})$; ${ }^{13} \mathrm{C} \mathrm{NMR}\left(\mathrm{CDCl}_{3}\right) \delta 164.4,142.3,123.2,72.0,67.5,52.7,32.0,27.6$; HRMS (CI) calculated for $\mathrm{C}_{8} \mathrm{H}_{11} \mathrm{NO}_{3}(\mathrm{M})^{+}$169.0739, observed 169.0740. 